To the Editors:

\title{
Thromboprophylaxis as routine for Sri Lankan surgical patients: where is the evidence?
}

I read with interest the leading article entitled "Deep vein thrombosis in Sri Lankans - time to take notice" by Sheriffdeen and Wijeyaratne (1). The authors state that they find it extremely difficult to understand why prophy. lactic measures have not been introduced in Sri Lankan operating theatres and wards as a policy in the light of overwhelming evidence for thromboprophylaxis. I firmly believe that clinical decisions should, as far as possible, be evidence based. Evidence-based medicine is the ".....explicit, judicious and conscientious use of the best available evidence...." to make clinical decisions (2). The authors have failed to cite a single publication from Sri Lanka to substantiate their claim for routine thromboprophylaxis in high risk groups.

Every surgeon has had patients who die post-operatively despite an apparently successful and even curative operation. Whatever we may like to think, these patients represent surgical failures. A common cause of unexpected death after surgery is pulmonary embolism (PE). The most reliable method of diagnosing fatal PE is by careful postmortem examination. In fact, autopsy studies have shown that PE is more common as a cause of post-operative death than is generally recognised, but no such studies are available in Sri Lanka. In the absence of autopsy evidence, such deaths are often ascribed to other causes. The evidence from studies of post-operative death in developed countries is remarkably consistent: if no prophylaxis is given, PE kills $0.5-1.0 \%$ of patients following major surgery (3). Deep vein thrombosis (DVT) can be regarded as the underlying origin of PE in nearly all post-surgical patients. If post-operative DVT could be prevented, most cases of PE could be avoided. This is the rationale for thromboprophylaxis in high risk patients in developed countries.

The clinical diagnosis of DVT is unreliable. Up to $50 \%$ of patients have no symptoms or signs in their legs, and only $50 \%$ of patients with leg symptoms are found to have DVT on further investigation. Most cases of postoperative PE, including many fatal cases, occur in the absence of any clinical indicator of an underlying DVT. Accurate assessment of the presence of DVT requires objective investigation. Venography, the gold standard for the detection of DVT, is a time-consuming and cumbersome technique for investigator and patient alike. ${ }^{125}$-labelled fibrinogen is simple and reliable, and is the technique most commonly employed in epidemiological studies and clinical trials. ${ }^{125}$ I-fibrinogen studies show that almost all postoperative deep venous thrombi start to form during surgery. Thus, early postoperative mobilisation cannot be expected to have a significant impact on the incidence of DVT. The Peradeniya group (1986) failed to detect a single case of postoperative DVT using ${ }^{12}$ I-fibrinogen in patients after open prostatectomy (4). In Europe, using the same techniques, a $40 \%$ incidence of DVT was reported after retropubic prostatectomy (1).

What is the high risk category these authors allude to? Age is the most important intrinsic risk factor and the nature of surgery is the most significant extrinsic risk factor. Any patient over $\mathbf{4 0}$ years of age undergoing major surgery should be considered at significant increased risk of venous thromboembolism. The risk of DVT is greatest with orthopaedic surgery: $75 \%$ after knee sugery, $60 \%$ after hip fracture surgery and 50 to $55 \%$ after elective hip surgery (3). The incidence of fatal PE after hip fracture sugery in $5.9 \%$ and after elective hip surgery $2.4 \%$ (3). Orthopaedic surgeons in Sri Lanka should conduct studies using ${ }^{125} \mathrm{I}$ fibrinogen to determine the incidence of DVT in their high risk patients and critically evaluate their 30-day mortality data, bearing in mind that in a substantial number of patients, death occurs after discharge from hospital, before they adopt a policy of routine thromboprophylaxis in high risk patients.

\section{References}

1. Sheriffdeen AH, Wijeyaratna M. Deep vein thrombosis in Sri Lankans. Ceylon Medical Journal 2001; 46: 3-5.

2. Sackett DL, Rosenberg WMC, Haynes RB, Richardson WS. Evidence based medicine: what it is and what it isn't. British Medical Journal 1996; 312: 71-2.

3. Bergqvist D. Postoperative thromboembolism: frequency, etiology, prohylaxis. Berlin: Springer-Verlag, 1983.

4. Sarath Kumara KA, Ratnasena BGN, Herath KB, Ranatunga PCA. The incidence of postoperative deep vein thrombosis in Sri Lankan patients. Proceedings of the Annual Scientific Sessions of the College of Surgeons of Sri Lanka, 1986.

Serozsha A S Goonewardena, Urological Surgeon, National Hospital of Sri Lanka, Colombo. 